the causes which predispose to tuberculosis, and must aim at avoiding or removing them. It is in this part of the general scheme of prophylaxis that the medical practitioner has so great a share to perform. The question of hereditary predisposition is here important. Your President in his opening remarks expressed what I suppose is the popular and usual opinion when he said that one could hardly note the way in which tuberculosis attacks certain families without recognising some potent hereditary influence. Granting this I do not place hereditary influence in the forefront of predisposing causes. My view, which I expressed before the Royal Medical and Chirurgical Society in 1894, is that the offspring of parents who are constitutionally unsound, whether from tuberculosis or any other cause, possess less resisting power against disease of all kinds, including tuberculosis. In 1000 families from about the same station in life I found that there was only an excess of 9 per cent. in the incidence of tuberculosis on the children of phthisical parents over that on the children of non-phthisical parents and that this excess might be explained by the former living with their consumptive parents-i.e., with an infective individual. The fact that the daughters-who remain longer at home-were chiefly attacked in these families, whilst the sons were most often affected in those families where the parents were nonphthisical gives support to this view. But whatever opinions we may hold on this point we shall all agree that it is only a predisposition which is inberited and not the actual disease. If this predisposition can be corrected, or sources of infection avoided, tuberculosis may be prevented. The children of consumptive parents should be kept from too close proximity to such parents. If it is the mother who is tuberculous the infant must not be suckled by her, and in any case the children should not share the parents' bed. To remove the predisposing constitutional weakness we must see that the early life is passed under the most favourable hygienic conditions. We must give attention to the feeding and clothing of the infant, to the hygiene of the nursery, especially as regards ventilation and sunlight; to the food, exercise, and hours of work of the schoolboy; and to the selection of occupation and place of residence for the adult. Acquired general predisposition may result from severe or prolonged illness, from depressing emotions, from worry, or from overwork and unhygienic surroundings. Hysteria and long-continued anæmia may pave the way for tuberculosis, measles and influenza are frequent predisposing causes, and in these ailments there is of ten local predisposition produced in the lungs, in addition to the general want of resisting power. Where full return to health is delayed after these illnesses, it is advisable to examine the chest carefully. I have elsewhere ${ }^{1}$ drawn attention to the pulmonary affections which may lead to phthisis, such as bronchitis and pneumonia, and bave already mentioned some local predisposing conditions in the fauces and pharynx. We are all aware that some strain or other slight injury generally precedes tuberculosis of a joint. Recognising the conditions which render an individual specially liable to suffer from tuberculous infection we should be on the alert for the first warning of disease in those who are thus predisposed, and by early treatment we shall be able not only to save our patient much suffering but to prevent much danger to others.

In conclusion, I must briefly refer to the rexed question of notification. On theoretical grounds I bave no doubt that notification is desirable, as I suggested before the International Congress of Hygiene in 1891, but it is in the application of the principle that diffculties occur. Instead of the rigid quarantine which was formerly imposed on those arriving from infected ports in the case of cholera and other diseases, we now allow travellers to land and to proceed to their destination; but the registration of such persons allows of a certain supervision until the expiration of the incubation period allays anxiety or the occurrence of the disease necessitates special precautionary measures. So with the consumptive or other tuberculous individual, notification and registration would render supervision possible. Where the circumstances and habits of the patient will ensure the advice of a competent medical man and the probability that his directions would be carefully carried out no interference by the medical officer of health would be necessary, and the person would not feel any disadvantage from a

1 The Medical Press and Circular, April 20th, 1892, and The Hygienic Prevention of Consumption (London : Chas. Ginfinand Co.), 1893, p. 38 . supervision which was non-obtrusive. But all experience shows that amongst the poorer members of society whose education does not help them to realise the importance of preventive precautions, whose habits are at variance with some of the prophylactic requirements, and whose means preclude the provision of others, more active supervision is essential if preventive measures are to be seriously attempted. Whilst work is still possible, such supervision might be sufficient, but in the advanced condition of the disease when work is impossible and when also the danger to others is greatest, removal to a hospital would be better for the patients and better for their families. For this purpose more special hospital accommodation would be needed than is now available. The provision of sanatoria also, which would receive only the earlier cases when cure is possible, cannot be omitted from any complete scheme for the prevention of tuberculosis.

\section{ON THE EXPERIMENTAL PRODUCTION OF HYDROSALPINX AND HYDROMETRA IN ANIMALS AND ITS RELATION TO HYDROSALPINX IN THE HUMAN SUBJECT.}

\section{BY C. J. BOND, F.R.C.S. ENG.,}

SURGEON TO THE IEICESTER INFIRMARY.

Is the course of an inquiry into another subject three years ago the following facts were observed, of which a preliminary note was published in the Journal of Physiology for February, 1898. In the following article I wish to draw special attention to such features as appear to throw light on certain corresponding conditions in the human subject. I was able, I think, at that time to fully establish the fact that if in the rabbit or guinea-pig and other animals a strictly aseptic abdominal section be performed and one ligature be placed round the Fallopian tube close to the uterine cornu and anotber just at the commencement of the tube and the continuity of the channel be thus blocked, in the course of a few weeks, and probably earlier, the portion of the tube between the ligatures distends with fluid secretion-in fact,

FIG. A.

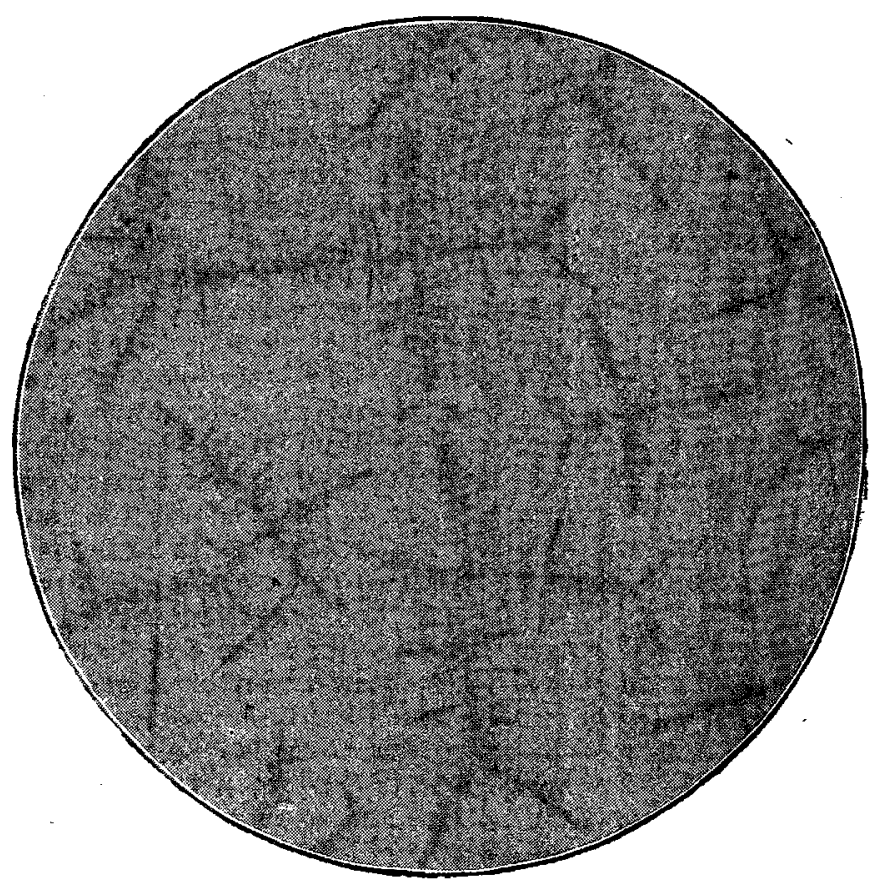

Fluid from human hydrosalpinx. Crystals of $\mathrm{NaCl}$ and another substance nature not determined.

a typical hydrosalpinx is produced. The fluid contents exactly resembles those found in a bydrosalpinx in the human tube produced pathologically. It is neutral or faintly alkaline in reaction, has a very low specific gravity, and is colourless, like water. It contains serum albumin, no globulin, a trace of an albumose, and some mucin. The 
chief mineral constituent is chloride of sodium and this is present in large quantities-a higher percentage, in fact, than in blood serum. The chloride of sodium crystallises out very rapidly on evaporation in feathery stars, and although there is no absolute constancy in the form of crystallisation certain forms seem more characteristic than others of certain species of animals. The fluid from a human hydrosalpinx also contained some crystals of a substance not found in the case of the lower animals. (Fig. A.) These, though somewhat resembling cholesterin, were not identical with that body.

Such is the character of the fluid which is secreted by the mucous membrane lining the Fallopian tube in animals and which collects in quantity when from any cause the outlet is blocked. If instead of ligaturing the tube the ligature be placed around the uterine cornu just above the junction of the cornua, then in animals the uterine cornu between the ligatures distends in the same way and a hydrometra results. Moreover, this uterine fluid has the same character as that derived from the tube and contains, like it, a large proportion of dissolved chloride of sodium. The fact that in some of the lower animals the secretion of the mucous membrane of the uterus resembles that of the tube is interesting and coincides with the slight degree of differentiation which has been reached anatomically, and apparently physiologically also, between these two portions of the oviduct. In the human subject, on the other hand, where differentiation has advanced further, while the natural secretion of the tubal mucosa is a watery fluid, that of the uterine membrane-at any rate, at the periods of menstruation-is of course a mixture of blood, mucus, and epithelial débris. Whether in the inter-menstrual periods any secretion occurs having any of the characters of a saline watery fluid we do not at present know. Such a secretion might occur, re-absorption preventing any external evidence. At any rate, in all cases of retained menses the retained fluid is a thick treacly liquid.

'There is, bowever, in animals one important difference in the effect of ligature of the tube and uterine cornu. If the tube be ligatured only at the uterine end and the abdominal ostium be left patent no distension occurs; secretion probably occurs as usual, but escapes into the peritoneal cavity and is there absorbed. It requires, in fact, closure of both

FIG. 1.

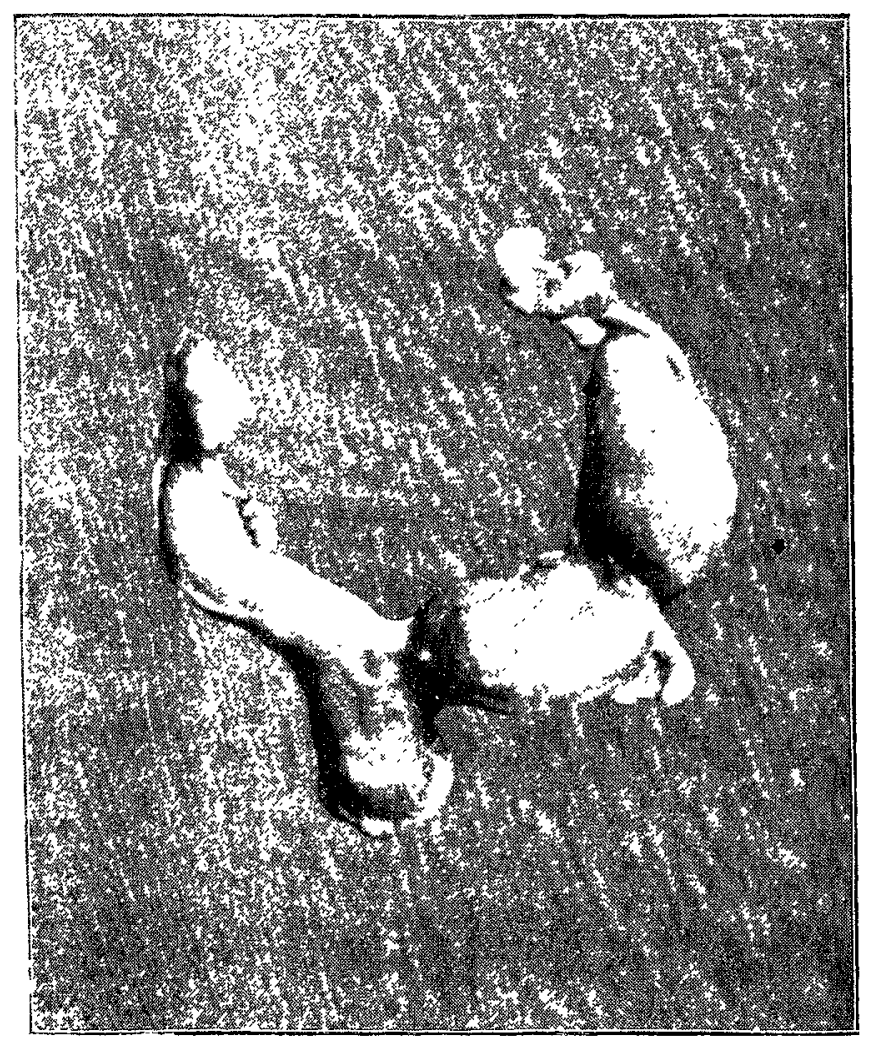

Guinea-pig. Showing ristension of right uterine cornu (hytrometra) aiter ligature. Distension occurs in the portion of the cormu between the ligatures $(A)$ and also in the portion hetween the upper ligature and the entrance of the Fallopian tabe (B). There is no distension of the tuhe (C).
Fig. 2.

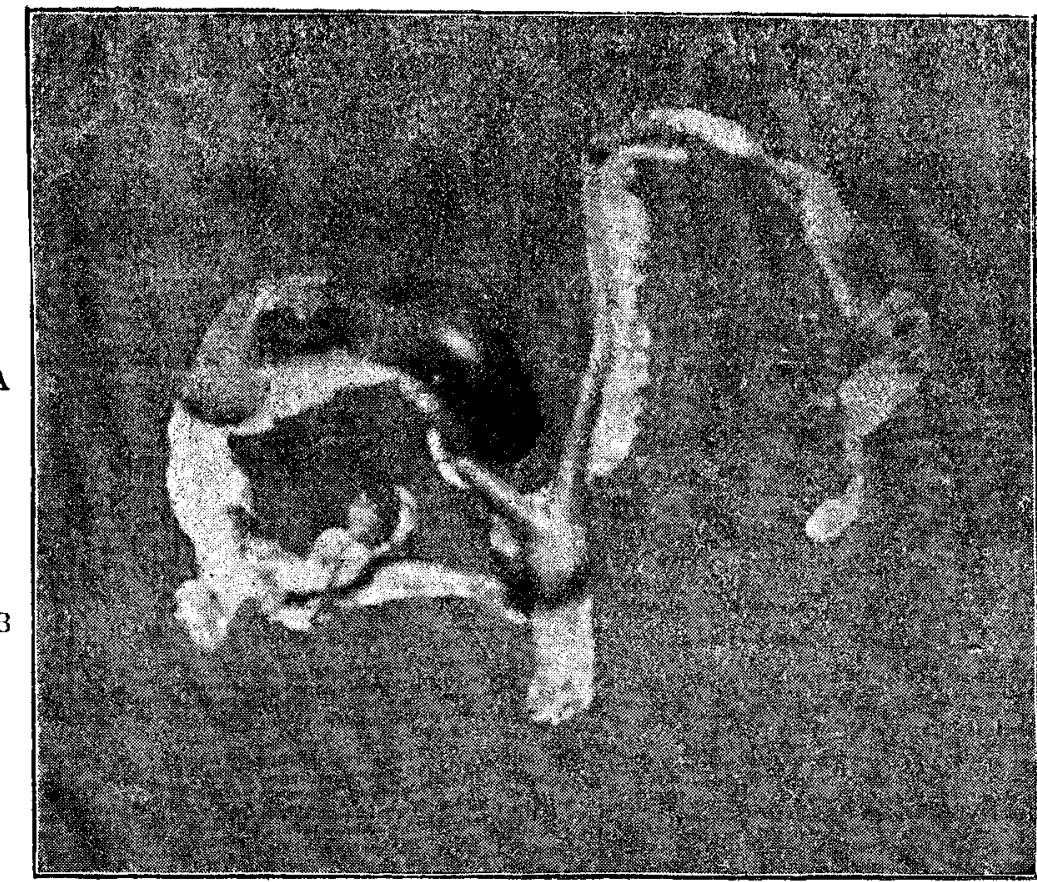

Rabbit. Showing distension of left nterine cornu (hydronetra). A is the portion of the cornu ahove the ligature. There is no dis tension of the Fallopian tube (B) cr mnligaturet cornu (C).

tubal ostia to produce a hydrosalpinx. If, on the otber hand, the uterine cornu be ligatured at any point down to the junction of the uterine cornua then the cavity above the ligature distends and hydrometra results (see Figs. 1 and 2); even though the communication between the uterus and the tube remains unobstructed any backward current is here prevented, although the rassage appears to remain clear in the other direction. This experiment throws some light on the fact that in the human uterus any distension of the organ by blood or fluids does not as a rule, except in cases of mechanical displacement or bacterial infection, cause backward cistension of the tubes. It does not, however, of course, affect the question of an infective inflammation of the endometrium spreading upwards into the tubes by means of bacterial agency; this unfortunately occurs only too frequently, neither is it necessarily opposed to the view that spermatozoa enter the tube. The following case of somewhat rare tubal malformation is interesting in this connexion. The vagina was absent in a young girl; an attempt was made by dissection to restore this canal; this unfortunately was followed by a septic endometritis which spread into the right tube, caus. ing a pyosalpinx; this was remored by abdominal section, and on the left side the following curious condition was found. The ovary was normal and the Fallopian tube in its abdominal and middle portions was quite normal; instead, however, of joining the uterine cavity the tube ended close to the uterine fundus in a cul-de-sac, no connexion existing $B$ between the two cavities. Lnlike the right side, the mucous membrane of the left or deformed tube was quite free from inflammation, no doubt owing to its disconnexion with the uterine cavity, thus, curiously, on the negative side, confirming the established fact of the origin of pyosalpinx in 1 infective endometritis. Moreover, this left tube was also quite free from distension, thus establishing in the human subject what I have already demonstrated in the case of animals-viz., that occlusion of the Fallopion tube at the uterine end alone does not produce distension by retained secretion or hydrosalpinx. It is also interesting to note that the curious malformation of the tube mentioned above, if bilateral, might be an overlooked cause of sterility.

It was this fact-namely, the absence of backward regurgitation from uterus to tube, even under pressure, in animalswhich influenced me in thinking that the menstrual fluid which $I$ found in the tubes in certain cases during menstruation in the human subject originated in the tubes as a true Fallopian menstruation. These observations on the occurrence of menstrual secretion in the Fallopian tubes were published in the British Nedical Jonirnal of June 4th, 1898. Micro-photosraphs of the secretion and of sections of the 
tubal mucosa were also published. After further observation I now think it more probable that in this, as in other cases, certain conditions are different in animals from those which occur in the human subject and that in a considerable number of cases in women in whom the uterus is retroverted regurgitation of the uterine menstrual fluid actually does take place into the tubes during the menstrual period, especially at the early part. It is, at any rate, suggestive that in most of the cases in which the fluid was found in the tubes there was great congestion of the fundus due to mechanical displacement. The cases of distension of the uterus from imperforate hymen, in which, as a rule, unless infection is present no tubal distension occurs, would seem at first sight to negative this hypothesis, but we must remember that no mechanical displacement such as retroversion is present in these cases. Since writing the above I have been able to demonstrate the passage of foreign particles (carmine) from the uterine cavity into the Fallopian tubes during menstruation, thus proving uterine regurgitation in some cases.

To return, however, to the main point of this paper. It has, I think, been clearly demonstrated that, at any rate in the lower animals, and in the human subject as far as the Fallopian tube is concerned, the mucous membrane lining the canal of the oviduct has a certain secretion of a peculiar and definite character. The following fact also shows that this uterine secretion is absent during pregnancy. A rabbit in which one uterine cornu was ligatured became pregnant directly afterwards in the other, or patent, cornu; this pregnancy pursued a normal course, and on killing the animal at term it was found that no distension of the cavity of the ligatured cornu or hydrometra had occurred, for although the tube was larger than in the non-pregnant state this was due to the hypertrophy of the mucous membrane which had occurred in the nonpregnant as well as in the pregnant cornu, though not to the same extent; in fact, it is impossible not to be struck with the similarity which a section of the uterine cornu of the pregnant rabbit bears to a section of the Fallopian tube. (Fig. 3.) An analogous condition is also

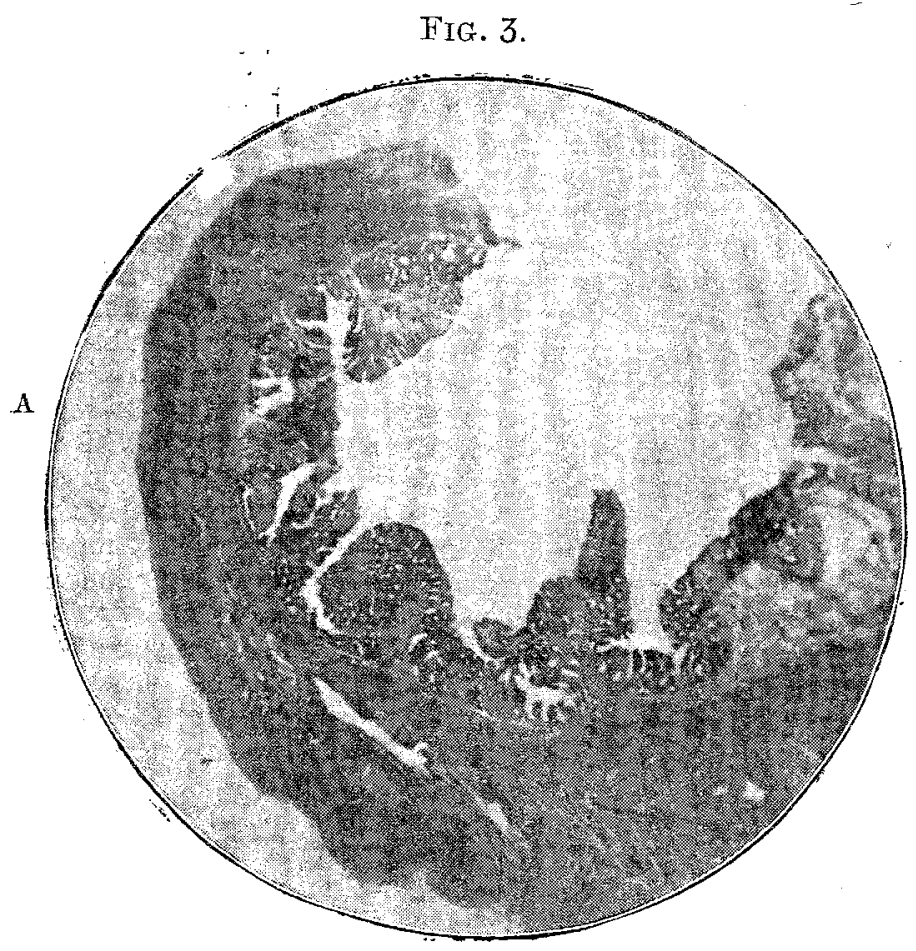

Rabbit. Hypertrophy of mucous membrane (decidual in ligaturel cornu uteri (A), pregnancy occurring in the other cornu.
No hylrometra.

present in the human subject when pregnancy occurs in one horn of a bicorned uterus. The accompanying illustration (Fig. 4) is from a case in which an extra-uterine or tubal pregnancy was present, in addition to a completely double uterus and vagina. In this rare condition, also, a well-marked decidua existed in both uteri, although of course neither contained a fœtus. Thus it appears that the uterine secretion is associated with the destructive processes which ordinarily go on in the generative canal in the female, and not with the constructive processes which take the form of increased growth of tissue and which are associated with pregnancy. Thus, one more example is added to the list of organs in which a secretion is formed and re-absorbed, although in this case, unlike the case of the ductless glands, the organ communicates with the exterior.

Finally, we must, I think, cease to regard human hydro. salpinx as the final or end condition in any case of infective inflammation of the Fallopian tube, it being really a mechanical distension by normal secretion in a tube in which the two openings have been closed ky inflammation. Pyosalpinx, on the other band, probably occurs when the infective process has affected the whole surface of the tubal mucosa, destroying its secreting powers and concerting it into an abscess cavity. It is also an interesting fact that the

FrG. 4.

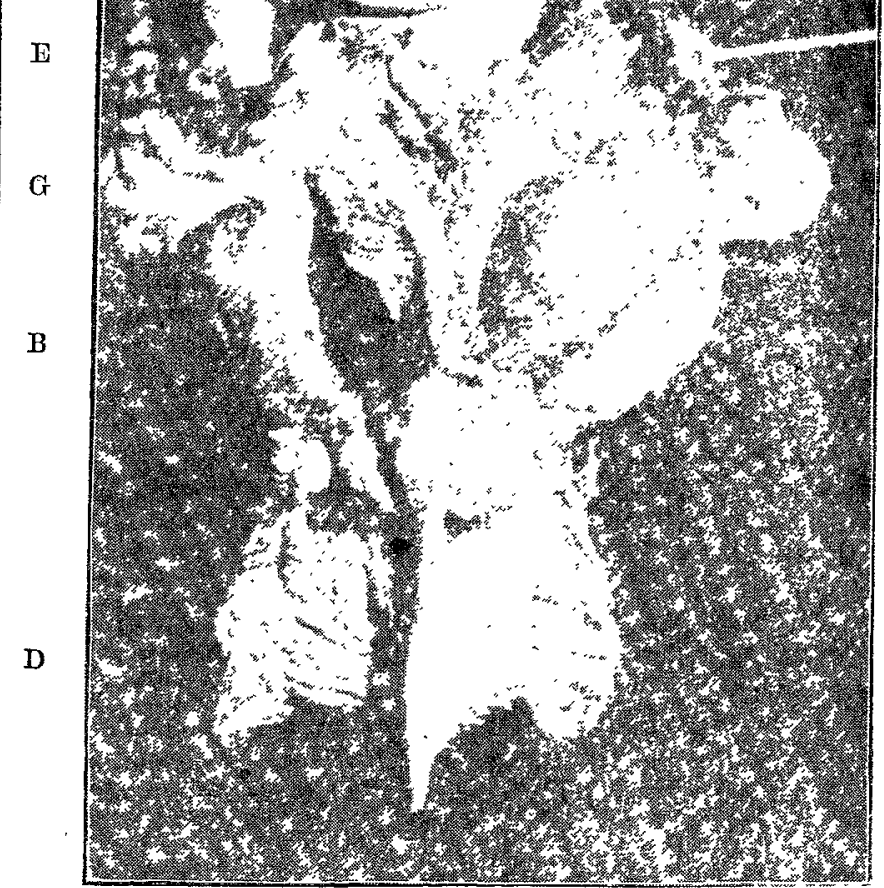

From photograph of doulle uterus and flouble vagina. A. Right uterus. C, Right vagina. B, Left uterus. D, Left vagina. $\mathrm{E}$, Sac containing foetus; hoth uteri contain lecidual membranes. F, Right ovary. G, Left ovary.

fluid found in a simple parovarian cyst closely resembles the fluid described above as occurring in distended Fallopian tubes. Thus it is of very low specific gravity, contains only a moderate amount of albumin, a little mucin, and is rich in chlorides. Bearing in mind the probable source of these cysts in a distended remnant of one of the efferent ducts of the paroöphoron or vestigial Wolffian body, organs which arise from the same embryonic source as the Müllerian duct, it is, under these circumstances, a matter of importance to find that the fluid contents in the two cases so closely resemble each other--a fact pointing to the origin of these parovarian cysts in a hyperdistension by a physiological fluid of a portion of the ducts in question.

Leicester.

The Medical Profession and the Friendly Societies: The Proposed BoARd of Conciliation.-At a meeting of medical officers of friendly societies in the district of Cardiff held on July 17th the following resolutions were carried: "That this meeting is of opinion that the profession should take the necessary steps to carry into effect in conjunction with the representatives of the friendly societies the proposal of the General Medical Council to form a board of conciliation." "That this meeting is of opinion that it is very desirable to form an association of friendly societies' medical officers in the Poor-law district of Cardiff." A provisional committee was appointed and Mr. T. Garrett Horder was asked to act as honorary secretary pro tem. 